\title{
Design of Mobile Terminal Software of the Intelligent Street Lamp Management System Based on GIS
}

\author{
Shuang Wang ${ }^{a}$, Yulin $\mathrm{Qi}^{\mathrm{b}}$, and Maowei $\mathrm{Li}^{\mathrm{c}}$ \\ School of North China Electric Power University, Baoding 071000, China; \\ a1053158201@qq.com,bqiyulin589@163.com,c1752006365@qq.com
}

Keywords: Street lamp management system, GIS, Baidu map API.

\begin{abstract}
Based on GIS, Baidu map API and Android SQLite database terminal software design of terminal management system which to Achieve user login, monitor the status of street lamps and fault repair and other functions, and ultimately the entire street lamp management system to achieve intelligent management of mobile terminals.
\end{abstract}

\section{Introduction}

With the continuous development of urbanization, the intelligent and wireless of street lamps are the mainstream development trend of street lamp management system. The continuous expansion of the number of street lamps make it difficult to control manually in the past. As a mobile terminal, the smart phone has an important application in some monitoring fields."Smartphone + street lamp terminal" mode has brought a new life for street lamp management. After the street lamp terminal software design is completed, the lighting quality of street lamp will be further improved to ensure the city's lighting rate and equipment intact rate.

GIS technology can combine the graphic management system and the data management system organically, and establish a street lamp management geographic information system by collecting, storing and analyzing the street lamp information. This design mainly uses Baidu map for development. Through the development of Baidu map API, it simulates the running status of the realtime street light and intelligent street lamp management mobile terminal software that reflects the failure information of the street light. The system simulates the connection of the database by specifying the location on the Baidu map Street lamp coverage by the street lamp management center on the street running status monitoring, through the mobile phone terminal to send a command to summon DC data, so the server from the street lamp terminal to collect DC voltage and current data sent to the mobile terminal, when the current is less than a certain The value is judged as a street lamp fault, thus real-time display on the map. So as to realize the effective monitoring of the entire street lamp management system by the management staff. The maintenance personnel can obtain the maintenance information through the smart phone terminal so as to provide strong protection for the timely repair.

\section{Key Technical Summary}

\subsection{GIS Program Development Environment}

Android is currently the most mainstream mobile terminal development platform, the underlying Linux-based, mainly used in portable devices such as smart phones and tablets. The overall structure is divided into four layers, in each layer has a number of independent functional modules. From the top to down are: Application, Application Framework, Android Runtime Libraries and Linux Kernel.

Android is an open development platform for third-party developers to provide a very broad, free space. Android platform phones seamlessly combine excellent Google services such as maps, email, search, etc. These services have become an important link between users and the Internet.

\subsection{Baidu Map API}

Baidu Maps API is a set of APIs based on Baidu Maps which provided free of charge to developers. It includes JavaScript, iOS SDK, Android SDK, Positioning SDK, LBS Cloud and other development tools and services. It provides basic map display, search, Positioning, inverse / geocoding, route 
planning, LBS cloud storage and retrieval capabilities for a variety of operating systems under the map application development. Baidu map is widely used in the country, providing floor maps, street view, satellite and other base map services, and supports a variety of browser access, better compatibility. Moreover, compared with Google map, baidu map provides comprehensive API and relatively small development difficulty. Therefore, we chose baidu map for development in this design.

\subsection{SQLite Database}

SQLite is a lightweight database that comes with Android. Because it consumes very little resources, SQLite is used by many embedded devices to store data and it currently supports mainstream operating systems such as Windows / Linux / Unix. Good compatibility. SQLite does not need to install and start the service process, it is just a suffix. DB database files, support for SQL statements, you can add, delete, change, check operations on the database.

\subsection{Server}

System uses the client server architecture, the server data forwarding function, through the address and port number, dual wireless terminals and mobile client can automatically connect to the server at work to achieve communication. Client and server can be connected using Web Services or Socket, Web Services more stable but cannot be two-way communication (with the server can take the initiative to connect, but cannot receive the server to send the alarm signal), and take up more memory; Socket technology structure instability, but after the establishment of the server and the client can send messages to each other on the initiative, its main features are low data loss, easy to use and easy to transplant. In summary, the system uses Socket technology for communication.

\section{The Overall System Design and Implementation}

The street lamp management system is composed of a street lamp terminal, a wireless network, a street lamp server and a smart phone terminal. The Android mobile terminal is connected with a server through a 3G / 4G network and a WIFI network, reads the status of the street lamp and sends corresponding instructions to the server, The control command is forwarded to the dual wireless terminal, and then transmitted to the street lamp terminal, to realize the monitoring of the street lamp by the mobile terminal. When detecting the failure information of the street lamp, the system displays the street lamp fault information to the Baidu map through the GIS technology .The corresponding street lamp covering on the map of Baidu will turn from trouble-free green to red, so as to intuitively tell the maintenance staff the information and the location of the troubled street light.

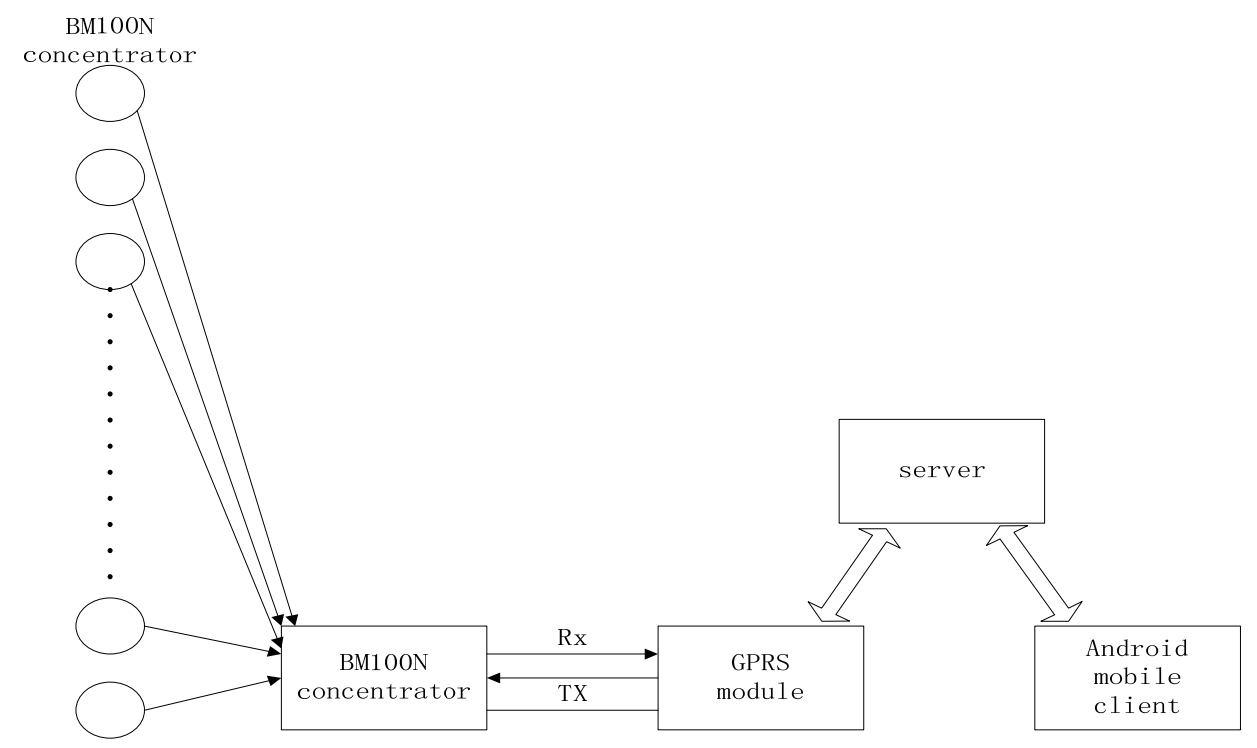

Figure 1 System Design Block Diagram 


\subsection{System Function Design}

System mainly through the mobile terminal software user login, street lamp status monitoring and street lamp fault repair and other functions. Users need to enter an account number and password when they log in to the system. When the user and password entered are within the system permission range, they can log in to the system. After logging in to the system, click the Summon DC button on the page to select a specific street light operation State to monitor, and thus jump to the location of the street where the display of Baidu map page to get real-time running lights information, when a street light fails, it will display the fault information on the map; when managers see the fault Information on the map, the timely arrangement of maintenance personnel to repair the faulty street light.

\subsection{User Login Module}

System login need to enter a user name and password, we set the administrator's username from 00-1023, and set a password for these users. The user name and password stored in the background database, when you enter the correct user name and password, will be allowed to log on to the system, and the corresponding operation. Login interface and Login successful interface are as shown in Figure 2 and Figure 3.

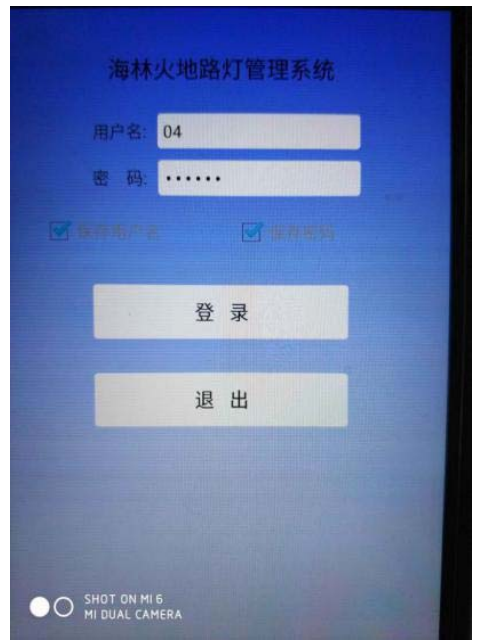

Figure 2 Login interface. Figure 3 Login successful. interface

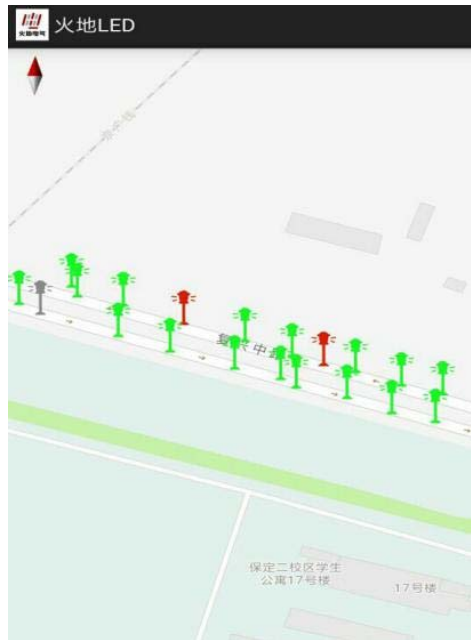

Figure 4 Street lamp monitoring interface

\subsection{Lamp Status Monitoring Module}

After the user successfully logs in to the system, he or she enters the login page as shown in Figure.3, enters the specified street number, and clicks the connection server, the system will connect with the server. When we click and call the DC data button, the system will send the corresponding instruction to the server, which will return the corresponding DC data through the server and jump to the Baidu map interface. By constantly requesting the server to realize the real-time monitoring of the status of the street light, The street light is gray when off and green during normal work, turning red when something goes wrong to alert managers to a prompt response. Street lamp monitoring interface shown in Figure 4.

\subsection{Equipment Maintenance Module}

When the street light breaks down, the manager will get the information by monitoring the change of the street light color and inform the relevant maintenance staff to repair the failed street light. When the maintenance personnel receive the fault information, they can reach the corresponding failed street light position through the map interface Street lights for maintenance. This can greatly save manpower and resources, improve work efficiency.

\section{Conclusion}

The application of mobile phone terminal software to the street lamp management system accelerates the intelligent management of the street lamp. The manager can monitor the running status 
of the street lamp, control the switch status of the street lamp and control the running status of the street light through a simple mobile terminal Run, ultra-low light operation), the most important thing is to jump to the Baidu map interface by clicking the call DC data button, and then shows the running state of the street lamp in the specified street, when the street lamp fails, you can find the fault and Timely repair of the faulty street light.Through the data forwarding function of the server, the realtime current and voltage data of the street lamp can be forwarded to the mobile terminal, and then the judgment can be made. When the current value is less than a certain value, it will be judged as a street lamp fault, and then the corresponding street lamp at the street lamp will change its color from faultless green to faulty red, remind the management staff to assign maintenance personnel to repair, greatly improve work efficiency, Reducing the cost of work.

The system has now entered the trial operation, greatly changed in the past rely on manual inspection to manage street lamps way to achieve the intelligent management of street lighting systems.

\section{References}

[1]. Wu Kunling. Design and Implementation of Urban Street Lamp Management System Based on Vector Map [D]. Xi'an: Xi'an University of Technology, 2014.

[2]. Lu Ting. Design of Remote Monitoring System for Street Lights Based on GPRS [D]. Dalian: Dalian University of Technology, 2009: 1-6.

[3]. Zhang Guowei. Design and Implementation of a Smart Street Lamp Control System Based on Google Maps [D] .Hefei: Hefei University of Technology, 2017.

[4]. Gong Chengying,Cao Bo.Design and implementation of urban lighting management system based on intelligent terminal [D]. Lanzhou: Lanzhou Institute of Technology, 2013 (5) .100-101.

[5]. Liang Guangrong, LI Hong. Virtual Campus Based on Baidu Map API [J]. Journal of Langfang Teachers College: Natural Science Edition, 2012 (12) .47-49

[6]. Zhang Binqiao, Yao Wei. Road and other integrated management system based on GPRS and GIS [D]. Hubei: College of Electrical and New Energy, Three Gorges University, 2013.135-136.

[7]. Song Aiping. Research and Application of Communication Methods in Urban Lighting Monitoring and Management System [J] .Journal of Illumination Engineering Science, 2009, 19 (3): 76-80. 